\title{
Neuroimmunological Registries in Germany
}

\section{(ㄷ) (1) $(9)$}

\section{Authors}

S. Thiel ${ }^{1}$, F. Leypoldt' ${ }^{2,3}$, L. Röpke ${ }^{4}$, K. P. Wandinger ${ }^{2,5}$, T. Kümpfel ${ }^{6}$, O. Aktas ${ }^{7}$, O. von Bismarck ${ }^{1}$, A. Salmen ${ }^{1,8}$, B. Ambrosius ${ }^{1}$, G. Ellrichmann ${ }^{1}$, G. Antony ${ }^{9}$, T. Dankowski ${ }^{10}$, A. Ziegler ${ }^{10,11,12}$, A. Stahmann ${ }^{13}$, C. Meyer ${ }^{13}$, K. Eichstädt' ${ }^{13}$, K. Buckow ${ }^{14}$, T. Meißner ${ }^{14}$, J. Thibaut ${ }^{15}$, L. Khil ${ }^{15}$, K. Berger ${ }^{15}$, R. Gold ${ }^{1}$, K. Hellwig ${ }^{1}$

Affiliations

1 Neurologische Klinik, St. Josef Hospital, Ruhr-Universität Bochum

2 Bereich Neuroimmunologie, Institut für Klinische Chemie, Universitätsklinikum Schleswig-Holstein Kiel/ Lübeck

3 Klinik für Neurologie, Universitätsklinikum Schleswig-Holstein Kiel

4 Klinik für Neurologie, Universitätsklinikum Jena

5 Klinik für Neurologie, Universitätsklinikum Schleswig-Holstein Lübeck

6 Institut für klinische Neuroimmunologie, Ludwig-Maximilians-Universität, München

7 Klinik für Neurologie, Medizinische Fakultät, Heinrich-Heine-Universität Düsseldorf

8 Universitätsklinik für Neurologie, Inselspital, Universitätsspital Bern, Universität Bern, Schweiz

9 Central Information Office (CIO KNP), Universität Marburg

10 Institut für Medizinische Biometrie und Statistik, Universität zu Lübeck, Universitätsklinikum Schleswig-Holstein, Campus Lübeck, Lübeck

11 ZKS Lübeck, Universität zu Lübeck, Lübeck

12 School of Mathematics, Statistics and Computer Science, University of KwaZulu-Natal, Pietermaritzburg, South Africa

13 MS Forschungs- und Projektentwicklungs-gGmbH, Hannover

14 Institut für Medizinische Informatik, Universitätsmedizin Göttingen

15 Institut für Epidemiologie und Sozialmedizin, Westfälische Wilhelms-Universität Münster

\section{Key words}

neuroimmunological registries, autoimmune encephalitis, neuromyelitis optica spectrum disorders, multiple sclerosis

\section{Bibliography}

DOI https://doi.org/10.1055/s-0043-108830

Neurology International Open 2018; 2: E25-E39

(c) Georg Thieme Verlag KG Stuttgart · New York

ISSN 2511-1795
Correspondence

PD Dr. Kerstin Hellwig

Neurologische Klinik

St. Josef Hospital

Ruhr-Universität Bochum

Gudrunstr. 56

44791 Bochum

k.hellwig@klinikum-bochum.de

\section{ABSTRACT}

Several neuroimmunological registries have been established in Germany during the last 10 years. The common aim is to investigate the course of different diseases, mainly under various therapeutic conditions, to identify predictive factors as well as the side effects of immunomodulating therapies. Six nationwide neuroimmunological registries will be presented in this article. The German Network for Research on Autoimmune Encephalitis (GENERATE) with more than 40 participating clinical centers and 570 documented patients (September 2016) collects data and biomaterials of autoimmune encephalitis with known and unknown antibodies. The registry coordinates and mediates between scientists and clinicians and acts as a platform for the development of guidelines and procedures. The neuromyelitis optica study group (NEMOS) has established a national registry for patients with neuromyelitis optica and neuromyelitis optica spectrum disorders. In addition to 22 academic hospitals, 17 regional hospitals and several practices are participating. Currently, 250 patients are enrolled. Moreover, NEMOS is now establishing a prospective patient cohort (NationNMO) within the competence network multiple sclerosis. Current research focuses on treatment strategies for relapses and interval therapy of neuromyelitis optica. The competence network multiple sclerosis has initiated a multi-center, prospective cohort study of patients with clinically isolated syndrome (CIS) or relapsing-remitting multiple sclerosis (MS) (NationMS), with the aim of long-term follow-up and clinical and paraclinical characterization of the patients. Between August 2010 and December 2014, 1,212 patients in 22 university und non-university centers were enrolled in the NationMS cohort. Standardized clinical data as well as biomaterial and MRI images were collected and stored. The German MS Society (DMSG, Bundesverband e.V.) established in 2001 a long- 
term project to obtain a consistent and reliable overview of MS patients in Germany. Since 2014 the registry has been revised comprehensively, with the main purpose of establishing a permanent data repository for healthcare research while ensuring the collection, storage and provision of data over decades and enabling the description of long-term outcomes. Currently, more than 170 German centers are participating and over 48,000 patients are enrolled in the registry. Since 2013 , the competence network multiple sclerosis has established the immunotherapy registry REGIMS with the objective to obtain information on incidence, type and characteristics of adverse events of new immunomodulating therapies for patients with multiple sclerosis or clinical isolated syndrome. As of January 2017 more than 700 patients have been recruited from 36 ac- tive centers. The German Multiple Sclerosis and Pregnancy Registry aims to obtain safety information of disease modifying drug exposure during pregnancy. In addition to safety aspects, disease course during pregnancy and postpartum and the identification of predictors of disease activity are investigated. During the last ten years 1,500 pregnant MS patients were prospectively enrolled in the registry and at least 250 new pregnancies are followed every year. These six registries make an important epidemiological and scientific contribution. Numerous colleagues from clinics and practice support these registries. The objective is the closer understanding of the disease course, the influence of therapeutic decisions and thus the improvement of counseling and care.

\section{Introduction}

Registry studies have the advantage of flexibility, longevity and acquisition of large amounts of data in a relatively short period of time compared to clinical trial interventions, without much loss of evidence. They are particularly suited to document rare diseases and pathologies as well as side effects, or to characterize special patient cohorts which cannot be included in clinical intervention studies (such as pregnant women). In the past 10 years several neuroimmunological registries have been created in Germany. Numerous neurologists in clinics and practices across the country support registry activities by referring relevant patients. In this article we would like to present six neuroimmunological registries for different disorders (autoimmune encephalitis, neuromyelitis optica spectrum disorders and multiple sclerosis), with different key subjects (progression, documentation of side effects, immunotherapies, desire to have children) ( $\triangleright$ Table $\mathbf{1}$ ). The registry structures will be presented as well as initial results and an outlook on further planned evaluations will be provided.

\section{Deutsches Netzwerk zur Erforschung autoimmuner Enzephalitiden (GErman NEtwork for Research on AuToimmune Encephalitis: GENERATE)}

\section{Goals}

Autoimmune encephalitis is a rare inflammatory disease of the central nervous system which, however, causes significant mortality, and above all morbidity and associated high socioeconomic costs [1]. In recent years, the field has experienced dynamic development by the pioneering description of synaptic autoantibodies as biomarkers and, in all likelihood, by disease-causing agents [2]. On the one hand, the discovery of new synaptic antibodies allows a persistent therapeutic and prognostic sub- and reclassification of autoimmune encephalitis syndromes [3]; on the other hand, the rapid growth of knowledge represents a diagnostic challenge for clinicians and scientists. Due to the rarity of the individual encephalitic syndromes, the more precise characterization of clinical phe- nomenology, prognostic implications, frequency of tumor association and response to therapies is a task that can only be particularly addressed by large clinical registries. German patients are distinctly underrepresented in current international cohorts [4]. The German Network for Research of Autoimmune Encephalitis (Deutsche Netzwerk zur Erforschung Autoimmuner Enzephalitiden) - GENERATE, founded in 2014, has the goal to improve treatment of autoimmune encephalitis. Achieving this objective requires research of risk factors, causes, mechanisms and progression of the disease. GENERATE should be considered as (1) clinical platform for networking healthcare centers in Germany, (2) point of contact for patients and relatives to find out regional contact persons, and (3) scientific platform for collecting clinical and paraclinical data and biomaterials to support scientific research approaches. For this purpose, a representative interactive homepage (www.generate-net.de) was created, an internet-based communication forum was set up for members, a national patient registry was established, central and decentralized biomaterial collections were organized, and diagnostic and therapeutic standards were developed.

\section{Structure}

The GENERATE registry contains a retrospective as well as a prospective cohort study, and was created as a multicenter study with 41 active centers at present, 35 of which are actively recruiting (see also $>$ Fig. 1 and $\mathbf{2}$ ).

The registry includes male and female patients of all ages (especially children and non-consentable patients after clarification to a legal guardian) with a clinical syndrome of autoimmune encephalitis (e. g., anti-NMDA receptor encephalitis, limbic encephalitis, brain stem encephalitis) (1) with demonstration of a specific autoantibody in the CSF and/or serum or a hippocampal antibody with unknown specificity or (2) clinical/paraclinical indication of immune-mediated genesis (response to immunosuppression, intrathecal immunoglobulin synthesis, and/or CSF pleocytosis, typical MRI changes such as bitemporal T2/FLAIR hyperintensities).

Included patients are locally pseudonymised and documented using a server-based database. Epidemiological, clinical and paraclinical data as well as therapies are recorded. All patients whose 
- Table 1 Overview of neuroimmunological registries.

\begin{tabular}{|c|c|c|c|c|}
\hline Registry & Disease & Focus & Number of cases & Goals \\
\hline GENERATE & Autoimmune encephalitis & $\begin{array}{l}\text { Symptomology, } \\
\text { treatment, progression }\end{array}$ & 570 & $\begin{array}{l}\text { Epidemiology, progression, prognosis and care } \\
\text { of patients with autoimmune encephalitis in } \\
\text { Germany }\end{array}$ \\
\hline $\begin{array}{l}\text { NEMOS/ } \\
\text { NationNMO }\end{array}$ & $\begin{array}{l}\text { Neuromyelitis optica } \\
\text { spectrum diseases }\end{array}$ & $\begin{array}{l}\text { Symptomology, treatment } \\
\text { and progression; } \\
\text { comparison with MS } \\
\text { (NationMS) }\end{array}$ & Approx. 250 & $\begin{array}{l}\text { Continuing education, consulting and support } \\
\text { of primary care physicians, patients and } \\
\text { relatives, multi-sector networking of treating } \\
\text { physicians }\end{array}$ \\
\hline NationMS & $\begin{array}{l}\text { Clinically isolated syndrome, } \\
\text { relapsing-remitting multiple } \\
\text { sclerosis }\end{array}$ & $\begin{array}{l}\text { Long-term observation of } \\
\text { initially untreated patients } \\
\text { in early disease stages }\end{array}$ & $\begin{array}{l}1,124 \text { in original } \\
\text { cohort; recruiting } \\
\text { (MRI cohort) } \\
\text { ongoing }\end{array}$ & $\begin{array}{l}\text { Detailed standardized clinical and paraclinical } \\
\text { data and biomaterial collection as the basis for } \\
\text { answering clinical and scientific questions, risk } \\
\text { and biomarker discovery and validation }\end{array}$ \\
\hline $\begin{array}{l}\text { MS registry } \\
\text { of the DMSG }\end{array}$ & $\begin{array}{l}\text { Multiple sclerosis, clinically } \\
\text { isolated syndrome }\end{array}$ & $\begin{array}{l}\text { Care monitoring and } \\
\text { (future) PRO }\end{array}$ & $\begin{array}{l}\text { Approx. } 10,000 \\
\text { patients/year } \\
\text { On average }>2.5 \\
\text { contacts/patient } \\
\text { per year }\end{array}$ & $\begin{array}{l}\text { Monitoring of care of persons with MS; } \\
\text { long-term observation of progression } \\
\text { (therapies); temporal changes in population; } \\
\text { patient inclusion, recruitment and research } \\
\text { platform }\end{array}$ \\
\hline REGIMS & $\begin{array}{l}\text { Multiple sclerosis, clinically } \\
\text { isolated syndrome }\end{array}$ & Pharmacovigilance & $\begin{array}{l}\text { To date } 700 \text { (in } \\
\text { development) }\end{array}$ & $\begin{array}{l}\text { Pharmacovigilance of MS medication; nature } \\
\text { and frequency of side effects to improve safety } \\
\text { of medications }\end{array}$ \\
\hline DMSKW & $\begin{array}{l}\text { Multiple sclerosis, clinically } \\
\text { isolated syndrome, } \\
\text { neuromyelitis optica }\end{array}$ & Pregnancy & Approx. 1,500 & $\begin{array}{l}\text { Safety of immune modulating therapies during } \\
\text { pregnancy and lactation, disease progression } \\
\text { during pregnancy and postpartum }\end{array}$ \\
\hline
\end{tabular}

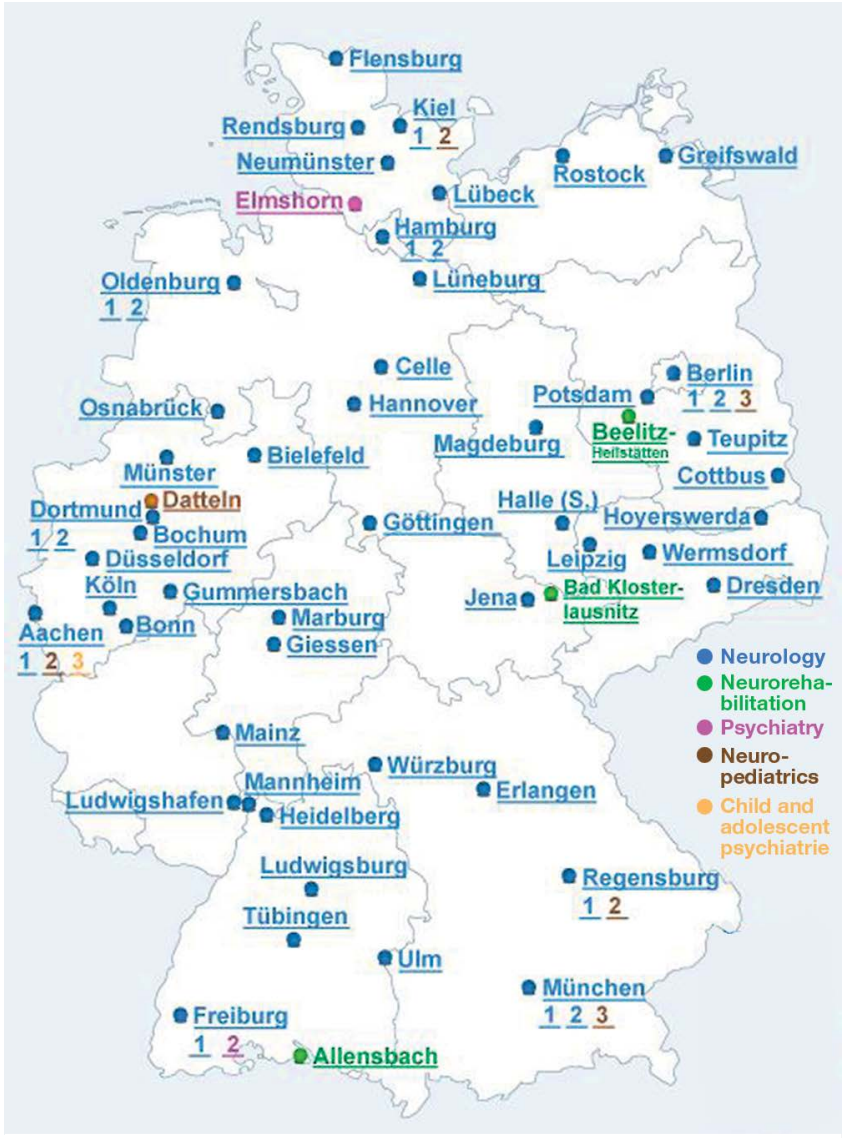

- Fig. 1 GENERATE centers in Germany, November 2016.

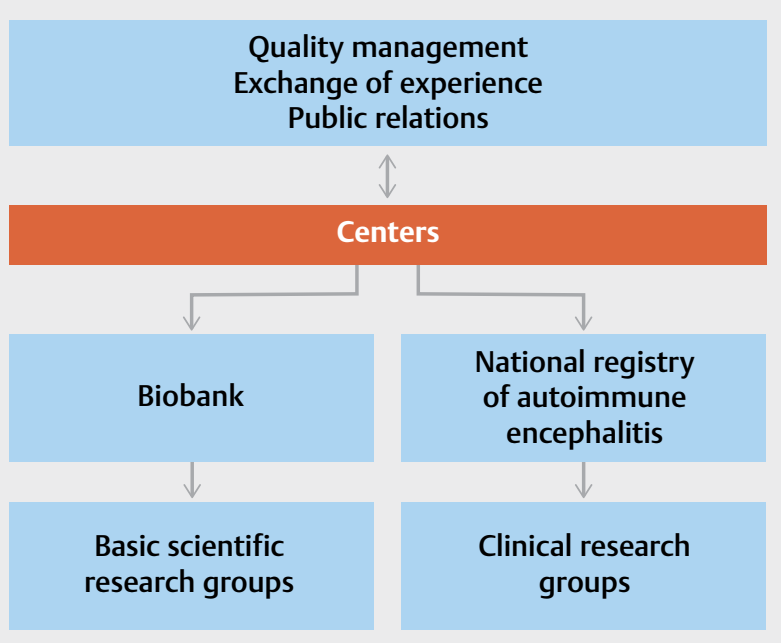

- Fig. 2 Structure of the GENERATE network.

symptoms began more than three months prior to inclusion are placed in a retrospective cohort, while patients exhibiting clinical symptoms within the previous three months prior to inclusion are grouped in a prospective cohort. Follow-up examinations are performed according to clinical criteria, generally every 3-6 months. Biomaterials (CSF, serum, DNA, CSF and blood cells) are preserved either centrally or decentrally and are available for scientific projects.

Scientific analyses are carried out (1) as registry-wide evaluations of clinical and epidemiological as well as prognostic research 
on behalf of the GENERATE study group, or (2) as single projects of individual centers with the possibility of an opt-out provision for each individual center. The structure and agreements within the network have been stipulated in a business regulation.

\section{Results}

As of September 2016, 570 patients have been documented in the GENERATE registry. An initial epidemiological evaluation is in preparation. An interim analysis shows that of 393 patients (April 2016), 61 (15.5\%) suffer from seronegative, and 332 (84.5\%) suffer from seropositive autoimmune encephalitis. Anti-N-methyl-D-aspartate receptor (NMDAR) encephalitis represented the most common seropositive group (34.9\%), followed by GAD-AK-associated encephalitis (15.4\%), followed by Lgi1 encephalitis (15.1\%) and CASPR2 encephalitis (7.5\%). Nine percent of seropositive autoimmune cases demonstrated prognostically unfavorable encephalitis with antibodies to intracellular antigens (paraneoplastic encephalitis) (unpublished results by Luise Röpke for the GENERATE network).

\section{Outlook}

Current research projects are investigating the genetic predisposition of autoimmune encephalitis, associated cerebrospinal fluid disorders and intensive medical complications, morphological image and neuropsychological aspects as well as therapeutic response rates. Likewise promising are an evaluation of long-term processes, investigation of patients with early escalation of immunotherapy as well as prognostic biomarkers in autoimmune encephalitis.

In February 2017, the non-profit GENERATE e.V. was founded as the organizer and sponsor of the network with the aim of continuing the existing structures. Expansion of the network to centers in Austria and Switzerland is in preparation.

\section{Registry for Neuromyelitis Optica Spectrum diseases}

\section{Goals}

Neuromyelitis optica (NMO, Devic syndrome), has been considered an independent disease after identification of a target antigen the water channel Aquaporin-4 (AQP4) [5-7], which should be differentiated from multiple sclerosis (MS) with respect to symptoms, prognosis and therapy [8]. Antibodies to AQP4 (AQP4-AK, synonym NMO-lgG) are detectable in NMO (but not in MS) in up to $80 \%$ of all patients [6,9]. However, NMO is a rare and, to date, little known disease pattern, which should be particularly distinguished from MS because of the therapeutic consequences. Thus, therapeutics used in MS, e. g., interferon- $\beta$ preparations [10] can have a negative effect on NMO [11]. In addition, the NMO-IgG/AQP-AK marker has led to new diagnostic criteria for NMO [12] or NMO spectrum disease, which can be defined when, in addition to a positive AQP4AK serostatus, there is evidence of a primary clinical criterion such as optic neuritis, myelitis, cerebral stem syndrome, dysphoric syndrome or an unusual cerebral lesion. An additional immunological heterogeneity is to be assumed with NMOSD: antibodies against the myelin oligodendrocyte glycoprotein (MOG) seem to play a rel- evant role in approximately $20-25 \%$ of NMOSD patients without evidence of anti-AQP4-AK.

Against the backdrop of these developments, the Neuromyelitis optica Study Group (German: Neuromyelitis optica Studiengruppe, NEMOS) was founded in Berlin in 2008, with the following main objectives:

1) Networking of regional, national and international clinical and scientific activities relating to NMO for medical colleagues and patients.

2) Complete (initially retrospective, in the course also prospective) recording of German verified and probable NMO cases in order to obtain important information on the epidemiology and the clinical course of the disease.

3) Collection of biomaterials from NMO patients (blood, cerebrospinal fluid) for scientific studies (e.g., comparison of different methods for detecting AQP4-AK, and as needed, development of new assays, genetic and immunological investigations).

4) Initiation of common therapy studies.

5) Development and publication of recommendations for diagnosis and treatment.

\section{Structure}

In 2008 NEMOS was established as a cross-sector network oriented toward clinical neuroimmunology designed specifically to address physicians active in outpatient and inpatient treatment and are interested in clinical and scientific issues relating to NMO. In total, more than two dozen clinics, medical centers and practices are incorporated into NEMOS ( $\triangleright$ Fig. 3); on the website www. nemos-net.org their activities are recapitulated by two NEMOS coordinators (currently T. Kümpfel, Ludwig-Maximilians-Universität Munich, and O. Aktas, Heinrich-Heine-Universität Düsseldorf). NEMOS also considers itself vertically open within the medical care hierarchy, thus providing opportunities for younger scientists to address relevant issues. Until recently, scientific inquiry, content coordination and public relations work were carried out on a voluntary basis without structural or financial support, thus priority was given to retrospective cross-sectional and longitudinal analyses based on existing documentation. Since 2016 NEMOS has been supported by the German Federal Ministry for Education and Research (BMBF) as a project within the Competence Network Multiple Sclerosis (German: Kompetenznetzwerk Multiple Sklerose, KKNMS) for the purpose of developing a national cohort (Nation$\mathrm{NMO}$ ) with standardized annual examination intervals. With regard to interaction with patients as well as with the wider medical profession/care structure, emphasis is placed on the website, contributions to patient forums and events, the involvement of patient organizations as well as information and training programs for neurologists and psychiatrists. The NEMOS website, www.nemos-net. org, maintained by the University Clinic Düsseldorf is certified according to the "Health on the Net" code of conduct for medical and health websites, directed toward patients and relatives, interested medical personnel as well as the NEMOS centers themselves.

The original NEMOS cohort accepted patients with NMO, isolated myelitis and optic neuritis with AQP4 seropositivity. The NationNMO cohort will include patients with NMOSD according to current diagnostic criteria (IPND 2015), excluding patients with MS or with isolated myelitis with MS-typical lesion distribution in a cranial MRI. 
The original NEMOS cohort recruited all NMSOD patients including those with only single examinations. In the NationNMO cohort there will be a prospective examination of patients with incidental NMOSD as those with prevalent NMSOD. Thus the NationNMO cohort will represent a formal subgroup of the NEMOS cohort.

The original NEMOS cohort relied on a non-time-limited collection of basal clinical data for diagnostics and therapy within the scope of neurological routine care, as needed (after identifying relevant clinical samples), as well as a subsequent expansion of data collection on an individual project basis.

In the NationNMO cohort, a prospective, standardized annual clinical and paraclinical data collection will be performed, designed to last 6 years, taking into account established parameters and scales: central collection of AQP4 and MOG serostatus; Expanded Disability Status Scale (EDSS); Multiple Sclerosis Functional Composite Score (MSFC); American Spinal Injury Association Impairment Scale (AIS); Multiple Sclerosis Inventory Cognition (MUSIC); Symbol Digit Modalities Test (SDMT); Beck Depression Inventory (BDI); Pain Detect, Functional Assessment of MS (FAMS); High and Low Contrast Visual Acuity; VEP; Optical Coherence Tomography (OCT).

\section{Results}

Public relations

Regarding public relations work, relevant stakeholders have been gradually approached over the past eight years and the understanding of NMOSD has been deepened as a rare but relevant disease. In concrete terms, it was possible to inform interested parties through patient events, and through the opportunity of direct contact via the NEMOS website, as well as providing support and advice to care providers, while directing them to local NMO specialists. Direct support opportunities for patients and relatives could be realized through participation in the medical advisory board of patient organizations such as Transverse Myelitis e.V. and involvement in relevant patient forums. Since its founding in 2008, NEMOS has regularly organized NMO-oriented symposia and workshops for physicians and other medical personnel at the annual conferences of the German Neurological Society (DGN), and has been recognized for this effort (Felgenhauer Prize 2014). The two consensus papers on the diagnosis and therapy of NMO should be considered a sign of these intensive coordinated efforts $[11,13]$, which currently are among the most cited overview articles in this area.

\section{Scientific activity and original work}

Since creating its database in 2008, NEMOS has established the basis for a number of recognized and internationally published papers on relevant aspects of the diagnosis and treatment of NMOSD. The first study investigated the initial cohort of $175 \mathrm{NMO}$ patients as to their demographic, clinical and imaging findings with regard to AQP4 serostatus [8]. In parallel, a subgroup of patients in this cohort was identified that was initially misdiagnosed as relapsing-remitting MS and were routinely put on a high-dose MS therapy with natalizumab [14]; despite this, the patients continued to exhibit persistent related disease activity $[15,16]$. This difficult diagnosis following a somewhat circuitous route was documented in an article on patients who initially underwent a spinal biopsy (which was in some cases not acceptably tolerated) for a suspect-

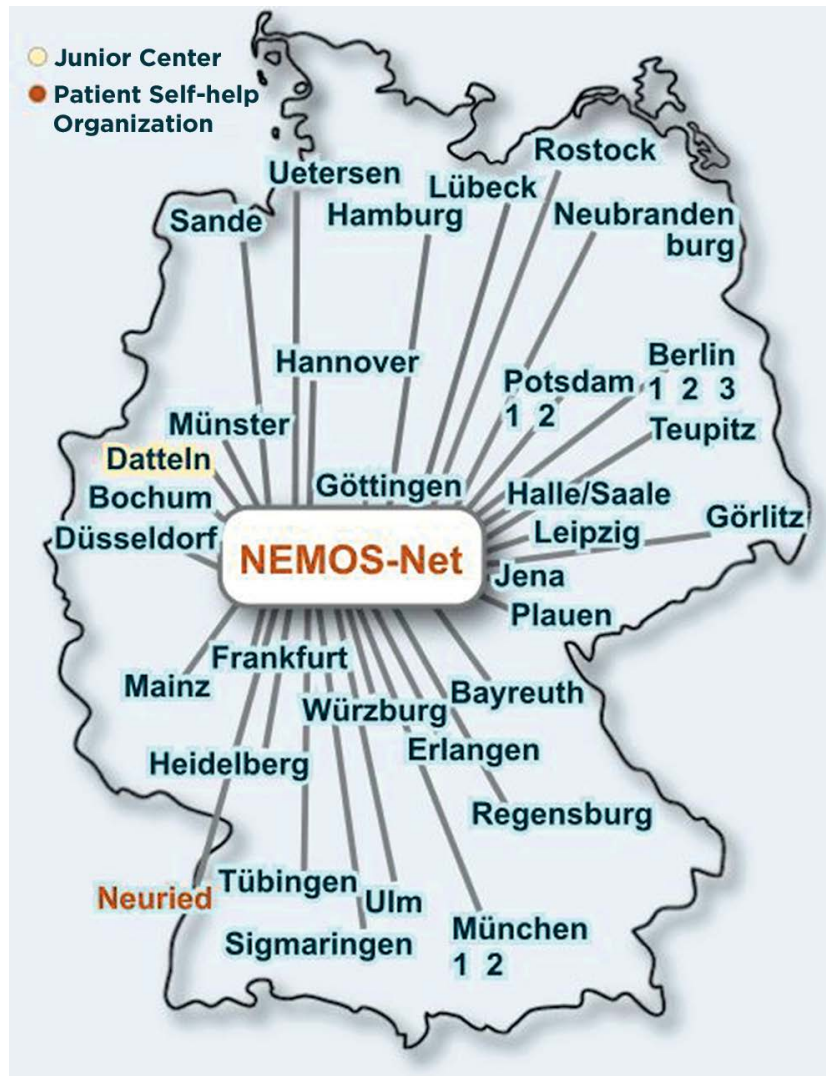

D Fig. 3 NEMOS centers in Germany, November 2016.

ed malignant spinal tumor, but who in the end had apparent tumefactive longitudinal extensive transverse myelitis (LETM) [17]. Furthermore, patients with unusual manifestations of NMOSD in late life stages were described using the NEMOS database [18]; additionally the influence of gender on clinical aspects of NMSOD was characterized [19]. In 2015 NEMOS was able to make a substantial contribution regarding acute therapy of NMSOD relapse by demonstrating the early importance of plasma separation/immune adsorption in particular for longitudinal extensive transverse myelitis (LETM) [20]. Finally, in 2016 NEMOS addressed the importance of a systematic approach to anti-MOG antibodies, describing clinical, imaging, demographic and immunological aspects [21, 22], as well as characterizing brain stem encephalitis which frequently accompanies this NMSOD variant [23].

\section{Networking internationally}

Right from the start NEMOS was engaged in scientific exchange with European and international NMSOD networks. In particular, the initiative of the Guthy-Jackson Foundation and the regular scientific conferences organized by this organization should be mentioned here. In this context, the path to a common global database for the comprehensive documentation of patients was outlined, and increasingly the influence of environmental and lifestyle aspects as well as genetic factors were discussed. It should be pointed out that in certain regions such as far east Asia NMOSD occurs much more frequently and does not have the status of a rare disease [24]. 
- Table 2 NationMS cohort study inclusion criteria (CIS = clinically isolated syndrome; RRMS = relapsing-remitting MS, $2 \mathrm{a}$ and $2 \mathrm{~b}$ alternatives).

\section{NationMS cohort study inclusion criteria}

1) Age $\geq 18$ years/male and female patients

2a) Diagnosis of CISwithin 6 months prior to inclusion

- fulfilling 3 of 4 Barkhof criteria [28]

OR

- fulfilling 2 of 4 Barkhof criteriaplus positive CSF findings (intrathecal igG production/oligoclonal bands)

OR

- meeting 2 of 4 Barkhof criteriaplus pathological visual evoked potentials (suggestive of MS)

OR

- fulfilling 2010 McDonald criteria [29] for RRMS

2b) Diagnosis of RRMS according to 2005 McDonald criteria [30] within 24 months prior to inclusion

3) Therapy-naïve for disease-modifying therapies (irrespective of relapse treatment)

- Table 3 NationMS cohort study exclusion criteria.

\section{NationMS cohort study exclusion criteria}

Primary progressive multiple sclerosis (PPMS)

Other progressive neurological disorders

Relevant contraindications for regular assessments (e. g., MRI)

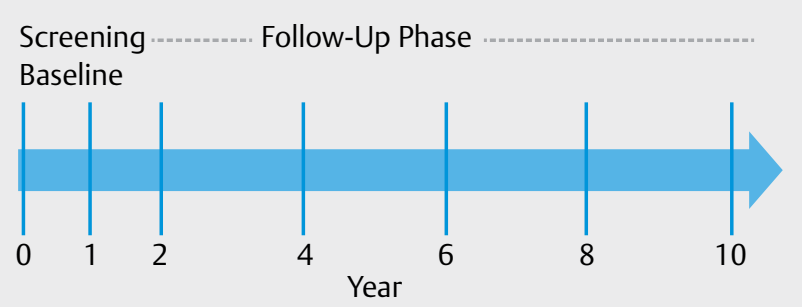

- Fig. 4 NationMS cohort study design.

\section{Outlook}

NEMOS has firmly established itself as a clinical-scientific network since its founding in 2008. The focus of current NEMOS activities is the expansion of a central patient database which allows simple input and maintenance of NMOSD patient data on the Internet, while adhering to legal and ethical regulations. This project is supported by the inclusion of NEMOS as a new partner in the BMBF-funded KKNMS (as "NationNMO cohort"), which is currently in its third funding phase. This grant is intended to support database-based, standardized patient documentation, fund a clini- cal-scientific comparison of the progression of the diseases and differentiation from MS as a direct reference disease while establishing a central biobank concept. In summary, NEMOS was embedded in already existing sustainable structures which made continuation of the NEMOS registry possible.

\section{Prospective Cohort Study of Clinically isolated Syndrome and Early Multiple Sclerosis Performed by the Competence Network Multiple Sclerosis (NationMS)}

\section{Goals}

MS is the most common chronic-inflammatory disease of the central nervous system in Central Europe. In 2010, based on data of the statutory health insurers in Germany, a total of 199,505 MS patients were identified, resulting in a prevalence of 289 per 100,000 population [25]. The estimated overall prevalence over the past three decades is 83 patients per 100,000 population [26]. MS disease frequency is higher the more northwards the country is located [27]. The validation of established risk factors, whether genetic or environmental, and uniform data of treatment monitoring algorithms can only be derived to a limited extent from German cohorts, since standardized data and biomaterial collections from large German multicenter surveys are largely lacking. This resulted in the initiative of the KKNMS to promote a German cohort study for patients with clinically isolated syndrome (CIS) and early MS, of which recruitment started in 2010.

\section{Structure}

NationMS is a prospective observational longitudinal cohort study (ethics vote Ruhr-University Bochum, no. 3714-10).

Inclusion and exclusion criteria are broadly defined in order to map the spectrum of the disease ( $\triangleright$ Table 2 and $\mathbf{3}$ ).

Since 2010, patients with CIS and early relapsing-remitting MS (RRMS) have been included in 22 university and non-university centers across Germany.

The study is designed for a total duration of at least 10 years, with the aim of long-term observation and clinical and paraclinical characterization of the patients. Study participants are examined in annual visits over the first three years and subsequently every second year ( $\vee$ Fig. 4). A deviation of 3 months from the scheduled visit date is accepted.

In addition to the collection of basic demographic and clinical data and patient questionnaires, standardized clinical and paraclinical assessment scales are implemented including the assessment of the domains of disability, cognition, depression, fatigue and pain. Following the consented standardized operating procedure (SOP), cranial MRI and asservation of biomaterial are performed at defined visits.

The comprehensive data protection concept is based on the generic data protection concepts of the Telematics Platform for Medical Research Networks (TMF), which have been coordinated with the Working Group for Science of the federal data protection authorities of the Federal Republic of Germany [31]. The coordinating center of the NationMS cohort (Department of Neurology, 


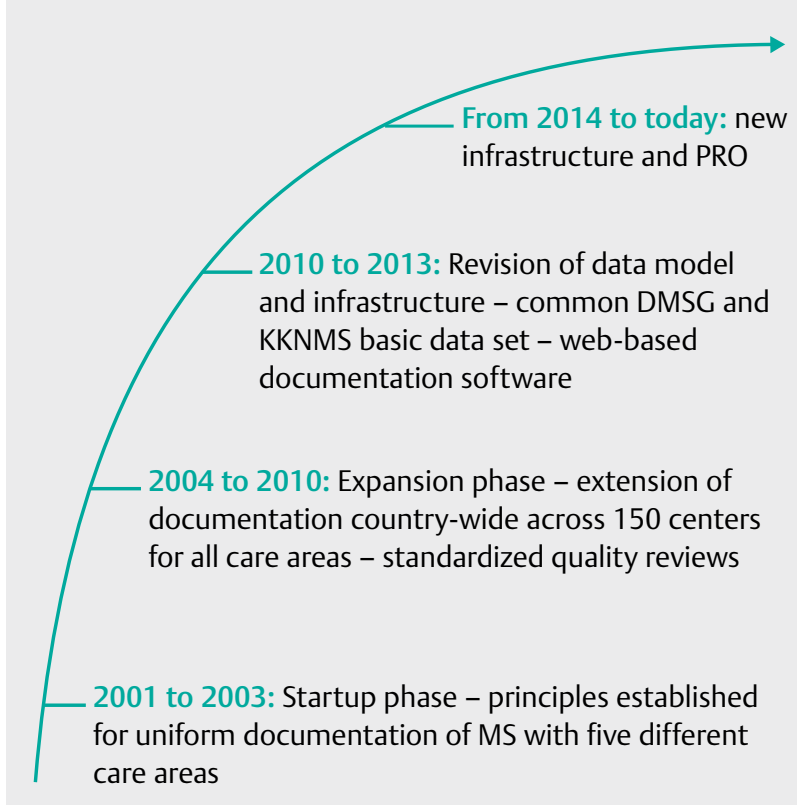

- Fig. 5 Developmental phases of the MS registry since founding 2001 until now [source-supplied illustration].

St. Josef-Hospital Bochum, Ruhr-University Bochum) performs standardized and independent monitoring and review of data.

\section{Results}

\section{Recruitment}

From August 2010 to December 2014, 1,212 patients were included in the NationMS cohort. Due to screening failure or incomplete data, 88 patients $(7.3 \%$ ) were excluded, resulting in 1,124 patients of the original cohort with evaluable records of the baseline visit ( $\triangleright$ Table 1). 960 patients have undergone their first follow-up examination (year 1 ). The subsequent follow-up examinations (year 2) are in progress in 2016. Since 2015, further recruitment has been carried out for an extended MRI cohort in centers with 3-Tesla MRI. So far, 172 patients have been included in this subcohort (as of 02-Nov-2016).

Analysis of the baseline visits show a gender distribution of 2.2: 1 (female $n=775$; male $n=349$ ). The diagnosis is CIS in $44.6 \%$ of patients $(n=501)$ and RRMS in $55.3 \%$ of patients $(n=622)$. Age at first clinical presentation (CIS and RRMS) is median 31.71 years (IQR 26.06-40.33), age at diagnosis (only RRMS) is 31.67 years (IQR 26.50-40.33).

\section{Scientific activities, original articles, public relations}

In addition to the evaluation of baseline clinical data, large-scale genetic projects have already been significantly supported with DNA material of the cohort and successfully published [32, 33]. Nine risk genes within the human leukocyte antigen (HLA) region and seven outside the HLA region were thus replicated within this German population [32]. In a further study, four new susceptibility genes were identified; their genetic products seem to be involved in epigenetic regulation [33].
In addition to scientific investigations, the collaboration within the KKNMS has led to consented recommendations addressing therapy monitoring for immunotherapies as well as the issue of the change of therapy [34,35]. This contributes to improvement of patient safety and possibly therapy optimization.

\section{Outlook}

The NationMS cohort is a unique large, multicenter and well-defined long-term study of patients with CIS and RRMS with standardized clinical and paraclinical assessment and biomaterial. At present, the detailed analysis of baseline data including demography and disease characteristics of this German MS cohort is ongoing. Further follow-up and ongoing recruitment are crucial for continued long-term observation.

Further current projects investigate relationships between clinical parameters and established environmental markers with the use of biomaterial. For example, an investigation of vitamin D status within the cohort as well as research of viral factors are running. Several groups are focused on the analysis of MRI parameters. Multicenter evaluations of large patient numbers are facilitated by the consensus MRI protocol.

The NationMS cohort facilitates ongoing and planned longitudinal and cross-sectional investigations. Due to the high number of participants, both subgroup analyses of defined patient groups and large-scale projects are possible. The availability of standardized data, biomaterial and MRI data will further expand over time. The NationMS cohort has thus special significance for the further acquisition of knowledge in MS. Hopefully, these results will rapidly find their way into clinical routine in order to improve diagnosis, therapy and care of MS patients in Germany; this has already begun as a result of collaborative work within the network via practice-related publications.

\section{The MS Registry of the DMSG}

Despite high and increasingly more expensive costs for the treatment and support of those suffering from MS, as well as the premature exit from employment of patients with MS, there is still little reliable information on prevalence of disease types or the care situation of persons with MS in Germany. Because of this, in 2001 the German Multiple Sclerosis Society (Deutsche Multiple Sklerose Gesellschaft, Bundesverband e.V., DMSG) established an MS registry as a long-term project in order to collect and analyze data obtained nationwide. The MS registry is managed by the MSFP-gGmbH ${ }^{1}$ as an independent entity under the sponsorship of the DMSG, and has continued to grow since its founding (see - Fig. 5).

\section{Goals}

The initial aim of the registry was to establish a uniform, reliable overview of MS in Germany for the first time, thus demonstrating potential needs of people with MS. For this purpose, data were collected and evaluated regarding a) disease course, b) distribution according to severity, c) employability, d) care aspects, e) regional distribution and f) therapies [36-42].

\footnotetext{
1 MS Forschungs- und Projektentwicklungs-gGmbH a $100 \%$ subsidiary of the DMS Stiftung of the DMSG, BV e.V.
} 


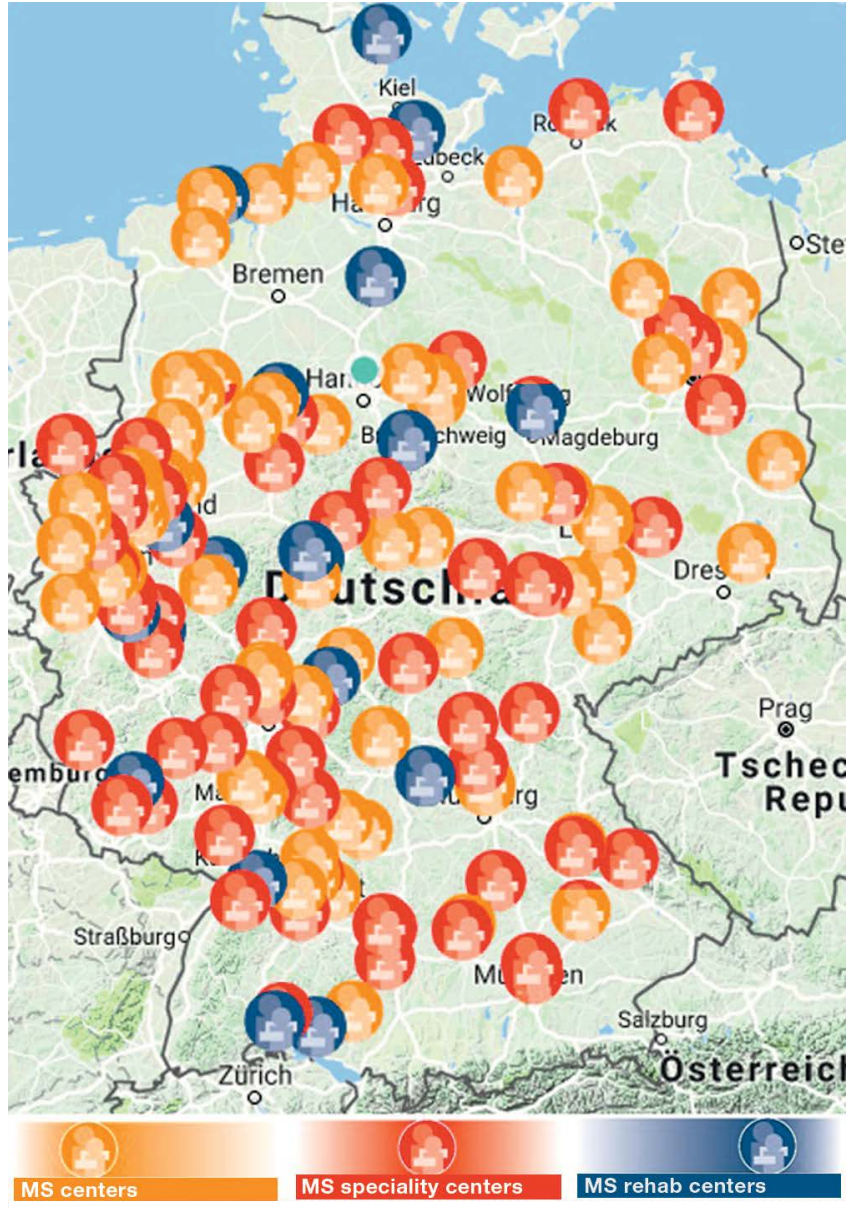

- Fig. 6 Distribution of centers in Germany as of 10/01/2016 [map data @ 2016 GeoBasis-DE/BKG (@2009), Google, Inst. Geogr. Nacional].

As part of a comprehensive revision in cooperation with the Institute for Medical Informatics of the University Medical Center Göttingen, the registry was methodically revised until 2014, both in terms of its infrastructure and the applied data set.

The current goal of the MS registry is to establish a permanent data repository for care research based on the over 10 years of experience. It will ensure the collection, storage and provision of data regarding MS patients over decades, and present long-term courses of the disease as well as monitor the care of those suffering from MS.

The revision laid the foundation for achieving participatory data collection [43], which includes both physicians and patients in data collection. Thus, the registry can support scientific research projects, for example, by using the data to generate hypotheses and to respond to these specific issues [44]. Furthermore, the registry will support the exchange of data with other national and international MS repositories. Such collaborations will more intensively compare regional aspects or opportunities of different health sys-

\footnotetext{
274 MS specialty centers, 80 MS centers and 19 MS rehabilitation centers (as of 01/13/2017) | map at https://www.dmsg.de/msregister/index. php?nav= zentren
}

tems, so that the requirements of individualized medical research can be better addressed.

\subsection{Structure}

The MS registry of the DMSG Bundesverband e.V. is a German Patient Registry with the following existing inclusion criteria:

- Written informed consent (after explanation by the center)

- Verified MS (according to McDonald or Poser) with selected disease course OR CIS

- Primary domicile in Germany

The MS registry has developed a nationwide network of MS centers including all areas ${ }^{2}$ of care (see $>$ Fig. 6 ) that recruit MS patients for the registry. These centers are awarded by the DMSG Bundesverband e.V. ${ }^{3}$. The broad network enables the included patients to be comprehensively documented crossing center and sector barriers, thus supporting the current focus on longitudinal observation of disease progression.

The centers involved in the MS registry are currently being migrated from decentralized data collection to a centralized, webbased documentation platform. The previously documented data are being transferred to the new infrastructure using a migration tool. After two years of transition, since the fourth quarter of 2016 , the new data set and the documentation in the web-based research database are obligatory for all centers. In addition to the web-based data entry, documentation can be performed in third-party systems which are connected via an appropriate interface to the research database.

The web-based solution made it possible to significantly reduce the IT requirements in the individual centers compared to using a decentralized software application. Only a PC or tablet connected to the Internet is necessary to access the documentation platform. Corrections or additions to the data set (for example, newly approved active substances and supplementary items) can now be performed centrally and made available to all centers simultaneously without delay. Same goes for updates of the platform.

In the web-based research database, the centers receive feedback on their documentation via checks of established value ranges, plausibility and cross-references in the event of discrepancies. These checks are supplemented by subsequent reviews of the total data pool. Queries regarding the documented data arising during monitoring are send out to the centers as integrated queries in the research database and can be answered directly there. An audit trail is also available and allows the tracking of changes to the data.

The data in the registry is collected prospectively and multi-centrically. It includes data from routine examinations of MS patients. Patient documentation is recurrent and consists of several modules that are filled out in:

- Baseline documentation: including basic data such as gender, age, initial symptoms and onset of the disease.

- and continuous follow-up documentations: comprising long-term data on the course of the disease and care of the respective patient.

\footnotetext{
3 https://www.dmsg.de/service/kliniken-praxen/dmsg-ausgezeichnete-zentren/
} 


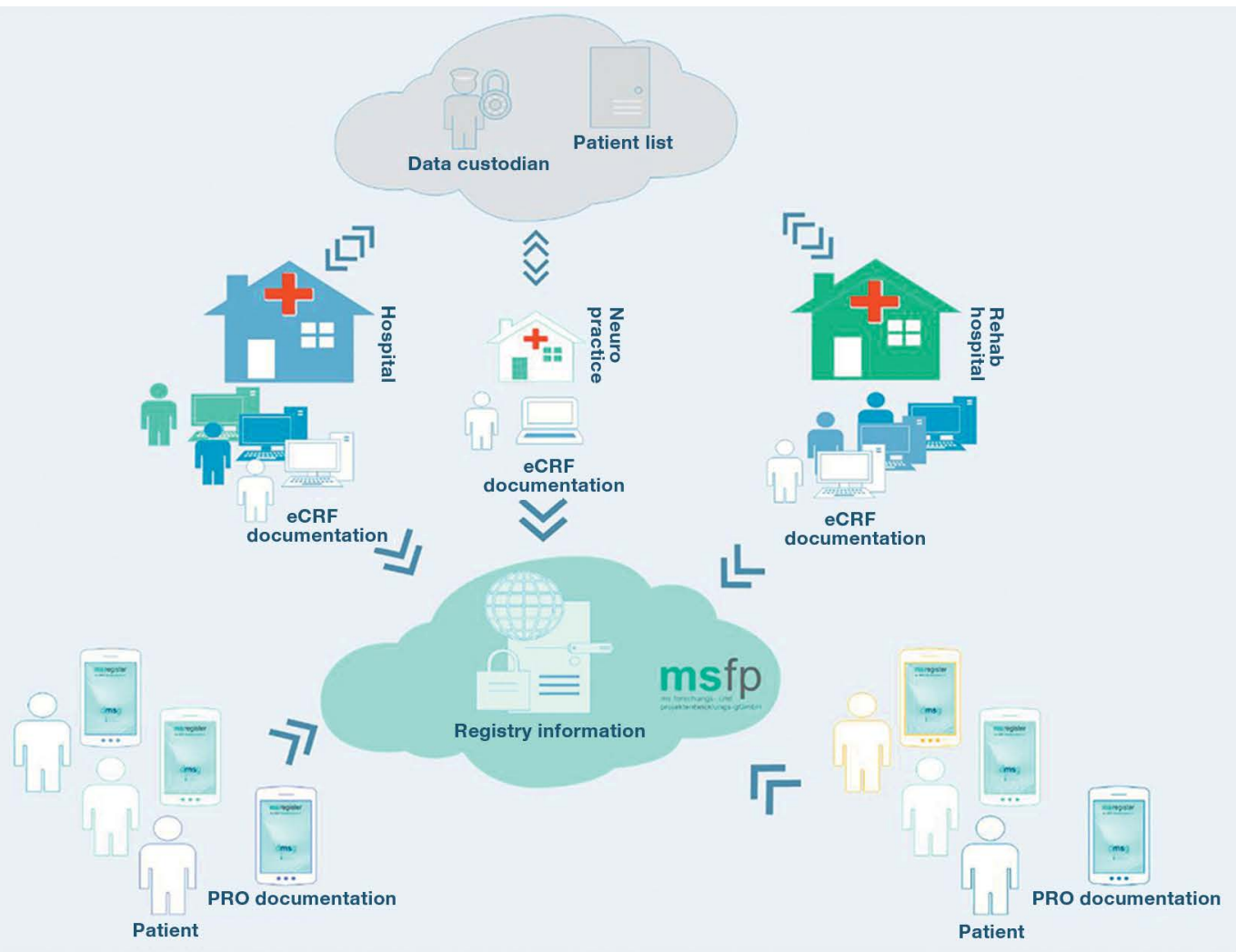

- Fig. 7 Inclusion of centers and patients in the documentation of the MS registry in relation to the underlying infrastructure [source-supplied illustration][44].

Currently the data to be documented is based on a data set jointly stipulated by DMSG and KKNMS, as well as the recommendations of the EUReMS project for the creation of a minimum data set, and includes registry-related additions. In an expansion module, selected centers document the current therapy as well as the history of the patients' therapeutic modifications. In the future, PRO data (e. g., Health Related Quality of Life, HRQoL) which are increasingly required to support scientific research questions and health policy will be linked to clinical data in the MS registry. During the revision, purely clinical data collection was expanded to include the patient's perspective. Variables of supplementary issues can be modularly included in the registry documentation, and the resulting data can be used together with the core data to respond to these questions.

The concept of the MS registry has been reviewed by various ethics committees. Patient participation in the registry is voluntary and can be terminated at any time. The MS registry is registered under the number DRKS00011257 in the German Clinical Trials Register.

The registry follows the structure illustrated in $>$ Fig. $\mathbf{7}$ which has been implemented in accordance with the generic data protection concepts of the TMF e.V. A strict separation of identifying data (IDAT) and medical data is maintained. A link between the pseudonymized medical and identifying data is only possible for the treatment centers in which the patient has given his/her consent to registering.

\footnotetext{
4 The following figures represent registry data as of September 2016
}

- Table 4 Registry population demographic data as of September 2016.

\begin{tabular}{|l|l|l|l|l|}
\hline \multicolumn{5}{|c|}{ Demographic Data } \\
\hline & $\begin{array}{c}\text { Age } \\
\text { (years) }\end{array}$ & $\begin{array}{c}\text { Duration } \\
\text { of MS } \\
\text { (years) }\end{array}$ & $\begin{array}{c}\text { EDSS } \\
\text { score } \\
\text { (median) }\end{array}$ & Female \\
\hline $\begin{array}{l}\text { Total data } \\
\text { (n=48,386) }\end{array}$ & $45.9 \pm 12.1$ & $12.8 \pm 9.7$ & 3 & $70.8 \%$ \\
\hline $\begin{array}{l}\text { Relapsing- } \\
\text { remitting } \\
\text { MS } \\
\text { (n=27,764) }\end{array}$ & $42.3 \pm 10.8$ & $10.5 \pm 7.9$ & 2 & $73.6 \%$ \\
\hline $\begin{array}{l}\text { Secondary } \\
\text { progressive } \\
\text { MS } \\
\text { (n=11,492) }\end{array}$ & $53.4 \pm 10.3$ & $20.5 \pm 9.8$ & 6 & $67.5 \%$ \\
\hline $\begin{array}{l}\text { Primary } \\
\text { progressive } \\
\text { MS } \\
(n=3,480)\end{array}$ & $55.5 \pm 10.8$ & $14.7 \pm 10.0$ & 5.5 & $58.2 \%$ \\
\hline $\begin{array}{l}\text { Clinically } \\
\text { isolated } \\
\text { syndrome } \\
(n=1,767)\end{array}$ & $37.8 \pm 11.2$ & $2.6 \pm 3.6$ & 1.5 & $69.7 \%$ \\
\hline \begin{tabular}{l}
$n$ \\
\hline
\end{tabular} & & & \\
\hline
\end{tabular}




\section{Results}

The MS registry answered for the first time important questions regarding the situation of patients with MS in Germany. Starting in 2005 , the initial results ${ }^{4}$ were published regarding the uniform data set resulting from the Germany-wide survey, providing information regarding distribution according to gender, forms of progression, symptoms, age at disease onset (average: 32.9 years), initial symptoms and time to diagnosis (average 2.7 years). See also excerpts of registry data in $>$ Table 4 . Furthermore, the type of care, the utilization (and discontinuation) of therapies and aids could be evaluated for the first time based on nationwide data collection. In addition, the registry provides data regarding the effects of MS on employment [37-41,45]

Based on the initial data obtained from the new registry infrastructure, the issue arising as a result of Petersen et al. [46] was answered: whether the proportion of patients with primary progressive MS (PPMS), who receive a therapy to modify the course of the disease contrary to the current guidelines, is also accounted for in the registry. Our evaluation showed a similarly high proportion of patients with PPMS receiving therapy. However, we were able to demonstrate that compared to earlier observations [40] the proportion has decreased [47].

In addition to cross-sectional observations, the MS registry offers the capability of long-term observations. The number of patients with coherent documentation over a 3 or 6 -year period is being continuously expanded and currently stands at more than 8,300 and 1,000 data sets. In $2015^{5}$ visits for more than 12,700 patients were documented. On average there were more than two consultations per patient in 2015.

The MS registry is open for research and collaborative projects ${ }^{6}$. For example, available data can be used during the planning phase of research projects to generate hypotheses, which, based on the new infrastructure and the modular data set can be answered as a supplementary question via the registry platform [44]. The MS registry has already successfully established data exchange at the national and European level.

\section{Outlook}

The available data are currently being used for additional issues, including "benign MS" and symptoms and provision of therapy, and are available for publication.

In the future, reports should regularly be provided to the documenting centers as an overview of their respective populations compared to the total population or the population range under care, thus supplementing the already available statistics and reports in the research database. A related pilot project is currently underway, providing the centers with a better overview of their patient data. In this way, for example, special characteristics (e. g., increased proportion of progressive patients) can be identified. In the past, such data have already been requested by the centers in order to answer, for example, inquiries regarding internal and external quality control.

Based on the experiences of a first pilot phase for the web- and app-based recording of PRO data [50], a concept is currently being

5 Data collection and assessment for 2016 is not yet complete.

6 Please direct related questions to the MSFP (msregister@dmsg.de). developed to involve patients in the registry documentation in the long term. A further field of action is the provision of data regarding the safety of disease-modifying therapies (DMTs). In the European network, the German MS registry is working with other MS registries to establish a system to provide the supervisory authorities with uniform data for the assessment of the safety of MS medicines.

\section{REGIMS Immunotherapy Registry}

\section{Goals}

The drug market for indication of MS is very dynamic since many new medications have been approved in recent years. New approvals concerned in particular the field of highly active immunotherapies [48]. Additional Phase III studies have been completed or are still underway [49] so that additional approvals can be expected in coming years. While frequent side effects are usually recognized prior to approval in Phase II and III studies, less common side effects are often not known at the time of approval because of limited patient numbers. An efficient post-approval pharmacovigilance system is therefore of great importance. Since the immune system is the focal point for many new medications to treat MS, long-term effectiveness as well as side effects of the therapy are relevant to ensure the efficacy and safety of the drug. The primary objective of REGIMS is therefore the establishment of a long-term immunotherapy registry for recording the incidence, type and characteristics of adverse events of current and future immunotherapeutic agents in MS therapy. Secondary goals include analysis of factors that increase the risk of drug side effects as well as those associated with good therapy adherence.

\section{Structure}

The registry includes patients with a confirmed MS diagnosis or CIS patients who are at least 18 years of age and who meet the following inclusion criteria: patients treated for the first time with an immunotherapeutic agent (de novo patients), or those treated with natalizumab, fingolimod, alemtuzumab, mitoxantron, teriflunomide, dimethyl fumarate, daclizumab or a drug released in the future regardless of duration of therapy; or those who have been treated with interferons, glatiramer acetate or azathioprine for a maximum of 36 months.

REGIMS is a multicentric registry of the KKNMS, which is coordinated at the Westfälische Wilhelms-Universität Münster. Participating centers are clinics, MS outpatient clinics and MS specialty centers that include patients in routine screening.

Data collection includes electronic documentation of medical parameters collected at participating centers by trained personnel as well as patient documentation in which the patient fills out a questionnaire. The basic medical documentation includes current and previous MS therapies, comorbidities, EDSS scores, relapses and any pregnancies. The patients are tracked by follow-up every six months. The medical follow-up documentation includes relapses since the last examination, changes in MS therapy, reasons for discontinuation of therapy, adverse and serious adverse events as well as pregnancies. In addition, the patient receives questions on 
the quality of life, therapy adherence and various lifestyle factors at 6-month intervals.

To reduce duplicate documentation and to take advantage of existing systems, three different input options are available for electronic documentation in the centers; these include secuTrial ${ }^{\circ}$, MSDS 3D $®$ as well as System $x 4 T$ which was specially developed for REGIMS. All REGIMS registry data are finally stored in the central KKNMS database. Due to their participation in REGIMS, all centers are also cooperation partners of the KKNMS.

Unwanted events of current and future immunotherapeutic agents are thus detected at an early stage (typically at occurrence) and reported to the manufacturers. The narrow time frames for the documentation as well as a parallel survey of both patient and physician guarantee this. This makes an overall picture of the disease and patient situation possible. In addition, centers and their patients have the option to participate in the biobank of the KKNMS. For this purpose, there is a separate consent procedure and a standardized method for blood collection and shipment. After acceptance, patients are monitored for the long term and surveyed at regular intervals. The registry therefore makes a key contribution to the drug safety of MS patients. The registry is registered under the number in the German Registry of Clinical Studies.

\section{Results}

After successful establishment of the immunotherapy registry since the start of the project in 2013, the number of new participating centers is steadily rising, and new patients are increasingly included in the respective centers. In addition, the semiannual medical follow-up surveys are carried out in the centers, allowing the description of medical information regarding MS therapy (including changes in therapy), the course of the disease, side effects and comorbidities. At the same time, every six months participating patients record their own experiences, such as quality of life, therapy satisfaction, therapy adherence and side effects from their personal perspective.

January 2013 was the initiation of REGIMS. As of 01/01/2017, 36 centers are active participants in REGIMS, with inclusion of over 700 patients. In the course of the coming months additional centers will join.

\section{Outlook}

Establishment of a pharmacovigilance database for MS medications is significant due to the approval of numerous new drugs to treat MS as well as the fact that additional medicines are in the approval process. An immunotherapy registry offers important advantages, especially with respect to the long-term effects of medicinal products. Country-wide recruiting of additional centers is currently in progress. In addition, collaboration with the MSBase international registry (www.msbase.org) [50] is in preparation as well as with the Swedish MS registry SMSreg [51] so that side effects can be recorded, assessed and reported on a broad basis.

\section{Deutsches Multiple Sklerose und Kinderwunschregister (DMSKW) (German MS and Pregnancy Registry)}

\section{Goals}

In recent years, only a few diseases have experienced such a great expansion of therapeutic possibilities as MS with the immunomodulatory therapies. Since the affected persons are primarily young women, the objective of the German MS and Pregnancy registry (DMSKW), founded exactly 10 years ago, is to obtain safety information regarding drug exposure during pregnancy. Since effective contraceptive measures are required for all DMTs not only during clinical trials, but also after market introduction, only limited data are available on the risks of exposure during pregnancy and lactation. To assess the safety of DMT exposure during pregnancy, the European Medicines Agency (EMA) requires at least 300, but preferably 1,000 prospective pregnancies with first trimester exposure. These large numbers of cases are only available for substances with long-term experience such as interferon- $\beta$ [52] and glatiramer acetate [53]. Data on more than 300 pregnancies was collected for natalizumab [54] and fingolimod [55] exposed pregnancies. There is insufficient experience with newer substances such as teriflunomide, dimethyl fumarate, alemtuzumab and daclizumab to evaluate the safety of exposure to these therapies during pregnancy.

In addition to safety aspects, the course of MS during pregnancy and postpartum as well as the identification of modifiable risk factors affecting relapse are of interest. The typical course of MS during pregnancy is well known, with a reduction of relapse rate up to $80 \%$ during the last trimester of pregnancy, followed by a significant increase during the first 6 months postpartum [56], and has been reproducibly confirmed multiple times [57, 58]. However, the influence of various different DMTs or the discontinuation of such therapies before a planned pregnancy on relapse during pregnancy and postpartum has not been sufficiently investigated yet. It is known that after discontinuation of natalizumab severe rebound relapses can occur $[59,60]$, and that they can also occur during pregnancy, despite its protective effect [61]. Whether this also applies to other DMTs which are used for highly active disease progression requires further examination. Some studies have shown that DMT therapy prior to pregnancy can reduces the risk of relapse during the first three months postpartum [62-64].

The aim of the DMSKW is to find the best possible treatment regimen for MS patients with the desiring to have children in order to minimize the risk for both, mother and child.

\section{Structure}

The DMSKW is a prospective cohort study of pregnant MS patients. Every year, approximately 250 pregnant women with CIS, clinically verified MS or NMO are admitted to the registry at any time during pregnancy. Similar to an embryotoxicological advisory institute, the registry is contacted by treating neurologists, gynecologists, pediatricians, MS nurses, but also by patients themselves with questions regarding pregnancy and MS, especially regarding dealing with MS therapies before, during and after pregnancy. The ma- 


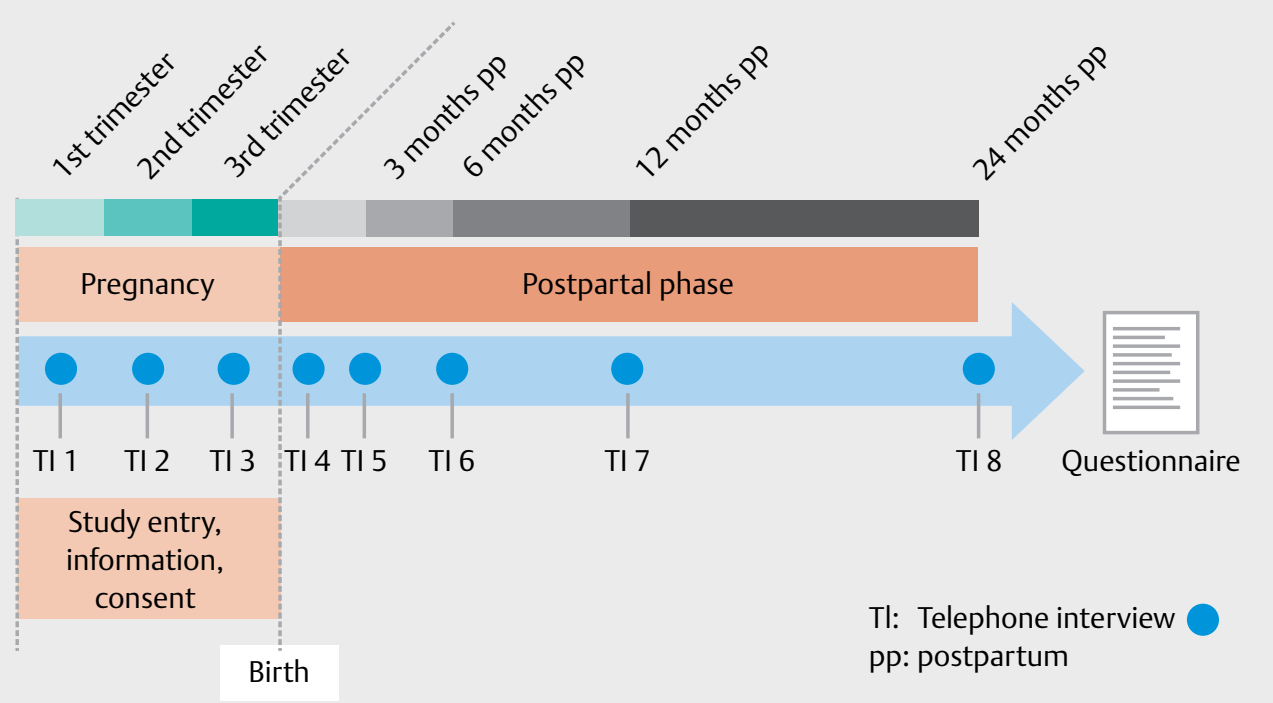

Fig. 8 Phases of the pregnancy registry during pregnancy and postpartum.

jority of women become aware of the DMSKW through advertisements in articles of magazines, publications on the homepage of the DMSG, or via the homepage of the DMSKW (www. ms-und-und-chinderwunsch.de) or they are actively recruited by their neurologists or MS nurses. Physicians or MS nurses are informed via publications in journals or advanced training courses. Data is collected in standardized interviews, generally by telephone. To avoid recall bias, interviews are conducted once during every remaining trimester after study entry and in months $1,3,6$, 12 and 24 postpartum (see - Fig. 8). In addition to basic characteristics of the mother, all data pertaining to drug exposure prior and during pregnancy is collected, as well as information relating to progression of the disease, pregnancy and birth. Progression of MS as well as child development are surveyed up to 2 years after birth. To limit information bias, data were obtained from official documents such as the maternity log, the child examination record as well as the vaccination record. In case of negative pregnancy outcomes or occurrence of MS relapses during pregnancy or postpartum, gynecologists, pediatricians and neurologists are contacted to verify the collected data. All congenital malformations are assessed and classified by a teratologist of the Mainz birth registry according to the current European surveillance of congenital abnormalities (EUROCAT) guidelines.

\section{Results}

In recent years, the DMSKW was able to answer many practically relevant questions by targeted data evaluation, and thereby to contribut the improvement of the care of MS patients desiring children.

Thus, further confirmation could be provided that exposure to glatiramer acetate [65], interferon- $\beta$ [66] or natalizumab [67] during the first trimester of pregnancy does not increase the risk of a negative pregnancy outcome, compared to pregnancies not exposed to DMTs. These results reflect the pharmacologically plausible safety of exposure to glatiramer acetate, interferon- $\beta$ or natalizumab during early pregnancy.
In a case series of 13 pregnancies of 12 women with highly-active MS treated with natalizumab during the third trimester, 10 out of 13 newborns had mild to moderate anomalies such as anemia, thrombocytopenia or leukocytosis [68].

Women who discontinued natalizumab therapy prior to a planned pregnancy suffered relapses more frequently during pregnancy than women who discontinued this therapy during the first trimester after confirmation of pregnancy [69].

Furthermore, the evaluation of women with moderate disease activity, who breastfed exclusively, partially breastfed or who did not breastfeed at all indicated that only exclusive breastfeeding reduced postpartal relapse activity [70], resulting in the hypothesis that the anti-ovulation factor, together with pronounced hormonal changes contributes to this effect. In addition it was observed that the reduction of relapse activity ends with the introduction of supplemental feeding.

In recent years the findings of the DMSKW have been able to improve the counseling and care of MS patients worldwide wishing to become pregnant. Thus the summary of product characteristics of natalizumab has included a statement regarding hematological abnormalities after exposure during the third trimester and resulting necessity for related monitoring, and most recently, the absolute contraindication for the use of glatiramer acetate (Copaxone ${ }^{\circledR}$ $20 \mathrm{mg}$ ) during pregnancy has been removed.

\section{Outlook}

Although the DMSKW has contributed substantially to the improvement of MS patients with the desire to have children, a number of questions remain unanswered such as the risk of interferon- $\beta$ or glatiramer acetate exposure during the second and third trimesters of pregnancy. In addition, the risk of exposure to newer therapies such as teriflunomide, dimethyl fumarate, alemtuzumab and daclizumab during the first trimester needs to be studied. Also of great interest is the risk of relapse after discontinuation of MS therapy prior to a planned pregnancy, especially with respect to women 
with highly active diseases. It may be feasible to reduce relapses during pregnancy and lessen the need for high doses of glucocorticoids by maintaining MS therapy until the onset of pregnancy. Low doses of prednisolone $(20 \mathrm{mg})$ are associated with a risk of cleft palate [71]. It is still unknown whether high dose treatments $(1000 \mathrm{mg})$ are associated with the risk of other abnormalities. It is also unclear whether exclusive breastfeeding also has a positive effect on the risk of relapse in women with highly active disease as well as the effects of DMT exposure during lactation on the infant. The influence of DMT exposure during pregnancy is also uncertain on the development and health of children up to the preschool age. To date, the DMSKW has collected data on the development and health of 232 small children in an unsystematic, cross-sectional observation. In the long term, however, it is planned to expand systematic DMSKW data collection to a 6-year follow-up.

\section{Conclusions}

Observational studies are an important part of epidemiological research. Registry studies have become an indispensable means of gaining knowledge, especially regarding data collection within specific patient groups, such as patients with very rare diseases (orphan diseases) or pregnant women. The most important advantages of these studies include the possibility to make statements about the progress of the disease in connection with therapeutic decisions and the related spectrum of side effects, multicentric collaboration as well as the support of registry work by contributions by colleagues in practice and research. Significant scientific questions can be answered through the collection of clinical data in combination with radiological or serological parameters.

Over the past 10 years the six registries presented in this article have established themselves across Germany, providing practice-related results not only for neurologists but also for those affected by neurological diseases. The findings based on these registries are almost unique and therefore of great importance especially for patients suffering from rare NMOSD or autoimmune encephalitis, but also for the special patient group of women with MS who wish to become pregnant.

All neuroimmunological registries presented here work closely with colleagues in research and practice, engage actively in public relations while making available relevant results at national and international conferences as well as publishing in medical journals. Consequently, these research groups not only provide a scientifically valuable contribution but also positively influence both the counseling and care of the affected patient groups.

\section{Acknowledgments}

In the name of the immunological registries GENERATE, NEMOS, NationMS, the MS registry of the DMSG, REGIMS und DMSKW, the authors would like to thank all their members for their motivated collaboration and recruiting, all patients and relatives for their willing participation, international cooperation partners for their collaboration, all contributing colleagues in research and practice as well as all supporters of the networks. The Competence Network Multiple Sclerosis is supported by the Bundesministerium für Bil- dung und Forschung (BMBF), funding codes $01 \mathrm{Gl0914}$ (Bochum), 01GI0916, 01GI1601G (Lübeck), 01GI1601B (Marburg) and $01 G 11602$ (Düsseldorf/Hamburg/Heidelberg for NationNMO/ NEMOS). The REGIMS registry is supported by the BMBF (funding code: 01GI1602E). Biogen has provided cofunding for natalizumab patients.

\section{Conflict of Interest}

F. Leypoldt works in an institute that offers commercial antibody testing and has received lecture honoraria from Grifols, Biogen, Roche, Teva and Bayer. L. Röpke has received support from the company UCB for one epilepsy educational event. T. Kümpfel has received remuneration and honoraria from Biogen, Genzyme, Merck-Sorono and Novartis. O. Aktas has received remuneration for consulting services from Biogen, Genzyme, Medlmmune and Novartis, as well as lecture honoraria and research grants from Bayer, Biogen, Genzyme, Novartis, Merck and Teva. A. Salmen has received remuneration and/or travel compensation from Almirall Hermal GmbH, Biogen, Merck, Novartis, Roche and Sanofi Genzyme. B. Ambrosius has received compensation for travel expenses from Novartis. G. Ellrichmann has received honoraria and research grants from Biogen Idec Germany, Teva, Novartis, Bayer Healthcare and Merck Serono. R. Gold has received consulting fees from Biogen and Teva as well as lecture honoraria and research grants from Biogen Idec Germany, Teva, Sanofi Aventis, Novartis, Bayer Healthcare and Merck Serono. K. Hellwig has received the honoraria and research grants from Biogen Idec Germany, Teva, Genzyme Sanofi Aventis, Novartis, Bayer Healthcare and Merck Serono. K. P. Wandiger, O. von Bismarck, S. Thiel, G. Antony, T. Dankowsik, A. Ziegler, A. Stahmann, C. Meyer, K. Eichstädt, K. Buckow, T. Meißner, J. Thibaut, L. Khil and K. Berger declare no conflicts of interest.

\section{References}

[1] Vora NM, Holman RC, Mehal JM et al. Burden of encephalitis-associated hospitalizations in the United States, 1998-2010. Neurology 2014; 82: 443-451

[2] Leypoldt F, Armangue T, Dalmau J. Autoimmune encephalopathies. Ann N Y Acad Sci 2015; 1338: 94-114

[3] Lewerenz ], Jarius S, Wildemann B et al. Autoantikörper-assoziierte autoimmune Enzephalitiden und Zerebellitiden. Nervenarzt 2016; 87: 1293-1299

[4] Titulaer M], McCracken L, Gabilondo I et al. Treatment and prognostic factors for long-term outcome in patients with anti-NMDA receptor encephalitis: An observational cohort study. Lancet Neurol 2013; 12: 157-165

[5] Lucchinetti CF, Mandler RN, McGavern D et al. A role for humoral mechanisms in the pathogenesis of Devic's neuromyelitis optica. Brain 2002; 125: 1450-1461

[6] Lennon VA, Wingerchuk DM, Kryzer TJ et al. A serum autoantibody marker of neuromyelitis optica: Distinction from multiple sclerosis. Lancet 2004; 364: 2106-2112

[7] Lennon VA, Kryzer T], Pittock S] et al. IgG marker of optic-spinal multiple sclerosis binds to the aquaporin-4 water channel. J Exp Med 2005; 202: 473-477

[8] Jarius S, Ruprecht $\mathrm{K}$, Wildemann B et al. Contrasting disease patterns in seropositive and seronegative neuromyelitis optica: A multicentre study of 175 patients. J Neuroinflammation 2012; 9: 14 
[9] Jarius S, Probst C, Borowski K et al. Standardized method for the detection of antibodies to aquaporin-4 based on a highly sensitive immunofluorescence assay employing recombinant target antigen. J Neurol Sci 2010; 291: 52-56

[10] Harmel ], Ringelstein M, Ingwersen J et al. Interferon-beta-related tumefactive brain lesion in a Caucasian patient with neuromyelitis optica and clinical stabilization with tocilizumab. BMC Neurol 2014; 14: 247

[11] Trebst C, Berthele A, Jarius S et al. [Diagnosis and treatment of neuromyelitis optica. Consensus recommendations of the Neuromyelitis Optica Study Group]. Nervenarzt 2011; 82: 768-777

[12] Wingerchuk DM, Lennon VA, Pittock S] et al. Revised diagnostic criteria for neuromyelitis optica. Neurology 2006; 66: 1485-1489

[13] Trebst C, Jarius S, Berthele A et al. Update on the diagnosis and treatment of neuromyelitis optica: Recommendations of the Neuromyelitis Optica Study Group (NEMOS). J Neurol 2014; 261: 1-16

[14] Aktas O. Natalizumab in clinical practice: Managing the risks, enjoying the benefits. J Neurol Neurosurg Psychiatry 2014; 85: 1181

[15] Kleiter I, Hellwig K, Berthele A et al. Failure of natalizumab to prevent relapses in neuromyelitis optica. Arch Neurol 2012; 69: 239-245

[16] Kleiter I, Hellwig K, Berthele A et al. Is it too early to predict the failure of natalizumab in NMO?-Reply. Arch Neurol 2012; 69: 1085-1086

[17] Ringelstein M, Metz I, Ruprecht K et al. Contribution of spinal cord biopsy to diagnosis of aquaporin-4 antibody positive neuromyelitis optica spectrum disorder. Mult Scler 2014; 20: 882-888

[18] Krumbholz M, Hofstadt-van Oy U, Angstwurm K et al. Very late-onset neuromyelitis optica spectrum disorder beyond the age of 75. J Neurol 2015; 262: 1379-1384

[19] Borisow N, Kleiter I, Gahlen A et al. Influence of female sex and fertile age on neuromyelitis optica spectrum disorders. Mult Scler 2016; 23: 1092-1103

[20] Kleiter I, Gahlen A, Borisow N et al. Neuromyelitis optica: Evaluation of 871 attacks and 1,153 treatment courses. Ann Neurol 2016; 79: 206-216

[21] Jarius S, Ruprecht K, Kleiter I et al. MOG-IgG in NMO and related disorders: A multicenter study of 50 patients. Part 1: Frequency, syndrome specificity, influence of disease activity, long-term course, association with AQP4-lgG, and origin. J Neuroinflammation 2016; 13 : 279

[22] Jarius S, Ruprecht K, Kleiter I et al. MOG-IgG in NMO and related disorders: A multicenter study of 50 patients. Part 2: Epidemiology, clinical presentation, radiological and laboratory features, treatment responses, and long-term outcome. J Neuroinflammation 2016; 13: 280

[23] Jarius S, Kleiter I, Ruprecht K et al. MOG-IgG in NMO and related disorders: A multicenter study of 50 patients. Part 3: Brainstem involvement - frequency, presentation and outcome. J Neuroinflammation 2016; 13: 281

[24] Pandit L, Asgari N, Apiwattanakul M et al Demographic and clinical features of neuromyelitis optica: A review. Mult Scler 2015; 21: 845-853

[25] Petersen G, Wittmann R, Arndt V et al. [Epidemiology of multiple sclerosis in Germany: Regional differences and drug prescription in the claims data of the statutory health insurance]. Nervenarzt 2014; 85 : 990-998

[26] Pugliatti M, Rosati G, Carton H et al. The epidemiology of multiple sclerosis in Europe. Eur J Neurol 2006; 13: 700-722

[27] Kingwell E, Marriott J], Jette $\mathrm{N}$ et al. Incidence and prevalence of multiple sclerosis in Europe: A systematic review. BMC Neurol 2013; 13: 128

[28] Barkhof F, Filippi M, Miller DH et al. Comparison of MRI criteria at first presentation to predict conversion to clinically definite multiple sclerosis. Brain 1997; 120: 2059-2069
[29] Polman CH, Reingold SC, Banwell B et al. Diagnostic criteria for multiple sclerosis: 2010 revisions to the McDonald criteria. Ann Neurol 2011; 69: 292-302

[30] Polman CH, Reingold SC, Edan G et al. Diagnostic criteria for multiple sclerosis: 2005 revisions to the "McDonald Criteria". Ann Neurol 2005; 58: 840-846

[31] Simon JW, Paslack R, Robienski J et al. Biomaterialbanken-Rechtliche Rahmenbedingungen.. Schriftenreihe der Telematikplattform für Medizinische Forschungsnetze (TMF), Bd 2. Berlin: Medizinisch Wissenschaftliche Verlagsgesellschaft; 2006

[32] Dankowski T, Buck D, Andlauer TF et al. Successful replication of GWAS hits for multiple sclerosis in 10,000 Germans using the exome array. Genet Epidemiol 2015; 39: 601-608

[33] Andlauer TF, Buck D, Antony G et al. Novel multiple sclerosis susceptibility loci implicated in epigenetic regulation. Sci Adv 2016; 2: e1501678

[34] Kolber P, Luessi F, Meuth SG et al. [Current aspects of therapy conversion for multiple sclerosis]. Nervenarzt 2015; 86: 1236-1247

[35] Klotz L, Berthele A, Bruck W et al. [Monitoring of blood parameters under course-modified MS therapy : Substance-specific relevance and current recommendations for action]. Nervenarzt 2016; 87: 645-659

[36] Flachenecker P, Zettl U, Götze U et al. MS-Register in Deutschland abschließende Ergebnisse der Pilotphase. Neurol Rehab 2007; 13: 193-200

[37] Flachenecker P, Stuke K, Elias W et al. Multiple-Sklerose-Register in Deutschland. Dtsch Ärztebl 2008; 105: 113-119

[38] Stuke K, Flachenecker P, Zettl UK et al. Symptomatology of MS: results from the German MS Registry. J Neurol 2009; 256: 1932-1935

[39] Khil L, Flachenecker P, Zettl UK et al. Patientenversorgung bei Multipler Sklerose: Vergleich der Basisdaten verschiedener Versorgungsstrukturen im Deutschen MS-Register. 82 Kongresses der Deutschen Gesellschaft für Neurologie mit Fortbildungsakademie. 2009;

[40] Khil L, Flachenecker P, Zettl UK et al. Update on the German MS Register: Immunotherapy and drug discontinuation. 25th Congress of the european committee for treatment and research in multiple sclerosis; 2009;

[41] Rommer PS, Buckow K, Ellenberger D et al. Patients characteristics influencing the longitudinal utilization of steroids in multiple sclerosis-an observational study. Eur J Clin Invest 2015; 45: 587-593

[42] Skierlo S, Rommer PS, Zettl UK. Symptomatic treatment in multiple sclerosis-interim analysis of a nationwide registry. Acta Neurol Scand 2017; 135: 394-399

[43] Stahmann A, Meyer C, Lee M et al. PRO-Dokumentation von Lebensqualitätsdaten im MS-Register der Deutschen Multiplen Sklerose Gesellschaft (DMSG). Bundesverband e.V. Forum der Medizin_Dokumentation und Medizin_Informatik - mdi. März 2015; 17: 18-19

[44] Stahmann A, Buckow K, Ellenberger D et al. Das MS-Register der DMSC als Plattform für die MS-Forschung. 88. Kongress der Deutschen Gesellschaft für Neurologie mit Fortbildungsakademie; 2015;

[45] Flachenecker P, Stuke K. National MS registries. J Neurol 2008; 255: (Suppl 6): 102-108

[46] Petersen G, Wittmann R, Arndt V et al. Epidemiology of multiple sclerosis in Germany. Nervenarzt 2014; 85: 990-998

[47] Stahmann A, Buckow K, Ellenberger D et al. The German MS Register: update on immunotherapy. ECTRIMS Online Library 2016; P1138

[48] Garg N, Smith TW. An update on immunopathogenesis, diagnosis, and treatment of multiple sclerosis. Brain Behav 2015; 5: e00362

[49] Michel L, Larochelle C, Prat A. Update on treatments in multiple sclerosis. Presse Med. 2015; 44: e137-e151 
[50] Butzkueven H, Chapman J, Cristiano E et al. MSBase: an international, online registry and platform for collaborative outcomes research in multiple sclerosis. Mult Scler 2006; 12: 769-774

[51] Hillert ], Stawiarz L. The Swedish MS registry - clinical support tool and scientific resource. Acta Neurol Scand 2015; 132: 11-19

[52] Romero RS, Lunzmann C, Bugge JP. Pregnancy outcomes in patients exposed to interferon beta-1b. J Neurol Neurosurg Psychiatry 2015; 86: 587-589

[53] Neudorfer O, Sandberg-Wollheim M, Coyle PK et al. The Glatiramer acetate pregnancy database. ECTRIMS Online Library 2015; 116357: P1507

[54] Friend S, Richman S, Bloomgren G et al. Evaluation of pregnancy outcomes from the Tysabri ${ }^{\circledR}$ (natalizumab) pregnancy exposure registry: a global, observational, follow-up study. BMC Neuro 2016; 16: 150

[55] Geissbühler Y, Vile J, Koren G et al. Cumulative data on pregnancy outcomes after exposure to fingolimod and in coparison with the general population. $32^{\text {nd }}$ Congress of the European Committee for Treatment and Research in Multiple Sclerosis (ECTRIMS); September 14-17, 2016; London, UK2016

[56] Vukusic S, Hutchinson M, Hours M et al. Pregnancy and multiple sclerosis (the PRIMS study): Clinical predictors of post-partum relapse. Brain 2004; 127: 1353-1360

[57] Salemi G, Callari G, Gammino M et al. The relapse rate of multiple sclerosis changes during pregnancy: A cohort study. Acta Neurol Scand 2004; 110: 23-26

[58] Hughes SE, Spelman T, Gray OM et al. Predictors and dynamics of postpartum relapses in women with multiple sclerosis. Mult Scler 2014; 20: 739-746

[59] Rigau V, Mania A, Befort P et al. Lethal multiple sclerosis relapse after natalizumab withdrawal. Neurology 2012; 79: 2214-2216

[60] Hellwig K, Gold R. Immune reconstitution inflammatory syndrome after withdrawal of natalizumab? Neurology 2011; 76: 1362-1363 author reply 3

[61] Hellwig K, Haghikia A, Gold R. Pregnancy and natalizumab: results of an observational study in 35 accidental pregnancies during natalizumab treatment. Mult Scler 2011; 17: 958-963
[62] Hellwig K, Haghikia A, Rockhoff M et al. Multiple sclerosis and pregnancy: Experience from a nationwide database in Germany. Ther Adv Neurol Disord 2012; 5: 247-253

[63] Paavilainen T, Kurki T, Parkkola R et al. Magnetic resonance imaging of the brain used to detect early post-partum activation of multiple sclerosis. Eur J Neurol 2007; 14: 1216-1221

[64] Fragoso YD, Boggild M, Macias-Islas MA et al. The effects of long-term exposure to disease-modifying drugs during pregnancy in multiple sclerosis. Clin Neurol Neurosurg 2013; 115: 154-159

[65] Herbstritt S, Langer-Gould A, Rockhoff M et al. Glatiramer acetate during early pregnancy: A prospective cohort study. Mult Scler 2016; 22: $810-816$

[66] Thiel S, Langer-Gould A, Rockhoff M et al. Interferon-beta exposure during first trimester is safe in women with multiple sclerosis-A prospective cohort study from the German Multiple Sclerosis and Pregnancy Registry. Mult Scler 2016; 22: 801-809

[67] Ebrahimi N, Herbstritt S, Gold R et al. Pregnancy and fetal outcomes following natalizumab exposure in pregnancy. A prospective, controlled observational study. Mult Scler 2015; 21: 198-205

[68] Haghikia A, Langer-Gould A, Rellensmann $G$ et al. Natalizumab use during the third trimester of pregnancy. JAMA Neurol 2014; 71: 891-895

[69] Spicher C, Thiel S, Klaaßen-Mielke R et al. Reversal of relapse pattern during pregnancy in women treated with natalizumab up to pregnancy (P2.096). Neurology 2016; 86 (16 Supplement):

[70] Hellwig K, Rockhoff M, Herbstritt $S$ et al. Exclusive breastfeeding and the effect on postpartum multiple sclerosis relapses. JAMA Neurol 2015; 72: 1132-1138

[71] Park-Wyllie L, Mazzotta P, Pastuszak A et al. Birth defects after maternal exposure to corticosteroids: Prospective cohort study and meta-analysis of epidemiological studies. Teratology 2000; 62: 385-392 